\title{
Research on the Marketing and Promotion of Boys' Love drama on Weibo from the Perspective of Fan Culture
}

\author{
Shubin Zhuang ${ }^{\mathrm{a}}$, Ruiyang Qin ${ }^{\mathrm{b}}$ \\ School of Humanities and Social Sciences, North China Electric Power University, Beijing, China \\ a120191170301@edu.cn, b120191170423@edu.cn
}

\begin{abstract}
In the Internet era, the market has put forward higher requirements for the drama marketing and promotion on Weibo. Besides, the IP craze such as fan culture and boy's love (BL) drama has brought new opportunities and challenges. The paper starts with a quantitative content analysis of the drama Guardian and The Untamed which are popular in recent years, looks into the influence of fan group and BL culture on Weibo's drama marketing and promotion based on the super group and official account, and puts forward corresponding strategies and suggestions. The study finds that producers, celebrities and fans are interconnected. Among them, fans take the navigating position whereas producers and celebrities are responsible for producing the content targeted at fan groups and exploring the methods of traffic monetization with grasping the emotional and economic model of fan culture. However, in this process, there are also problems such as capital and platform power misuse, the irrational activities. Therefore, more normative guidance of the BL adaptation culture and BL dramas needs to be put on the agenda.
\end{abstract}

Keywords: Fan Culture; BL Drama Adaptations; Traffic Realization; Weibo Marketing.

\section{Introduction}

In recent years, BL dramas have gone viral. The total view counts of Guardian reached 2.7 billion by the end of playing the final episode and the daily views ranked in the top third with the highest up to 100 million. Besides, the online discussion volume of Guardian got to 16.744 billion. All of them showed that the era of BL adaptation came. In terms of the The Untamed, it had been played more than ten billion times by the end of 2021. A month after the broadcast, the daily views still remained 200 million. As many as 1.63 million people scored the TV drama on Douban. (data source: Douban, Youku, Tencent, Weibo, etc.) It could be seen that the BL drama adaptations have reached a peak in the field of TV series. With the background of fan culture, the paper analyzes the marketing and promotion on Weibo from the perspective of BL adaptation culture, content production, and traffic monetization, trying to innovatively supplement the academic research on the marketing of TV dramas on Weibo in China.

\section{Literature Review}

\subsection{The Exploration of Youth Subculture in the New Media Context}

\subsubsection{Definition and Feature Analysis}

In the context of new media, Wu Heng pointed out that "due to the homogenization of network information, the information field that people focus on will be habitually guided by their own interests, so as to limit their life in a 'cocoon' where pieces of the same information gather together to spread in the network."[1] According to that, the paper believes that youth subculture is a way of communication and entertainment for young people as Internet "aborigines" to use the interaction and virtual space of Internet platforms to form positions for similar groups.

The characteristics can be summarized into three categories, namely interest-oriented, deconstructive and subversive, liquid and clustering. According to Examination of the Characteristics and Values of Youth Subculture of Fan Jiajun [2], it can be concluded that youth subcultures are connected with emotions. Driven by the media mechanism, young people are constantly finding partners with common interests to form groups with multiple emotional resonance. Moreover, based 
on Bakhtin's Carnivalization Theory, clustering is viewed as the action of young people who look for utopias in the new media context and the self-immersion within the highly interactive instinct herd mentality and shared emotions. With the New Tribe Theory, Chen Long and Li Chao (2021) also described the youth subculture as a new tribe on the level of a certain atmosphere and mentality [3]. Last, the liquidity refers to the young people playing different roles in different and even overlapping groups to meet the multidimensional value orientation.

\subsubsection{Analysis of the Existence of Youth Subculture}

According to the academic research on youth subculture, there are mainly internal difference extremism and external mainstream culture reduction. First, in the New Trend of Online Youth Subculture [4], Ma Zhonghong and $\mathrm{Hu}$ Liangyu defined the internal difference extremism as a phenomenon that sub-cultural groups differ in core emotions. Combined with Fan Jiajun's Examination of the Characteristics and Values of Youth Subculture [2], the paper analyzes the main reason for the differences into competitive relationship and interest's influence. Besides, the group mechanisms involving the competition in communication space and the content production effect can lead to difference. For example, the heatedly discussed "Xiaozhan's Fans and Fan-fiction Groups Incident". Second, it is a consensus in the academia that youth subculture has greatly reduced the mainstream culture. In the context of new media, youth subculture begins to get rid of the marginalized position and become more popular, whose values will affect the public imperceptibly, especially on the shaping of youth value orientation. In addition, the active development of youth subculture has seized the communication media, squeezed the space of mainstream culture, and reduced its discourse power.

\subsection{Traffic Monetization from the Perspective of Fan Culture}

\subsubsection{The Definition and Economic Analysis of Fan Culture}

Fan culture, emerging in the Internet era, is an organization form of fan culture in the context of new media with a core focusing more on traffic. With the continuous innovation of media form as well as the social media popularity, individuals used to be free and independent are connected in new channels. Well-structured fan groups with clear assignments are formed in the development to strengthen the connection and interaction with stars and carry out group normative activities containing content production and data operation. To put it simply, fan group culture is a strong circle communication mode and a inner-group operation mechanism generated by the transformation of fan culture in the context of new media, which has a strong structure and strict group behavior rules. The reason why it is classified into the research paradigm of youth subculture lies in its unique features of organization, operation, and industrial chains. To some extent, fan group culture is regarded as an emotional economy that has a complete organization and operation structure, giving birth to new digital circulation forms and new media culture industry. Based on the cross-field observation in the economic field, the academia has proposed that fan group culture shows a unique economic feature.

\subsubsection{The Traffic Monetization of Fans, Platforms, and Celebrities}

Fans, platforms and stars are interrelated and interactive in the marketing profit model. Fans, as the main source of content production, provide a large number of users, strong user engagement, and long viewing period, providing stars traffic as virtual currency that can exchange for resources. Platforms, as media, provide space for fan-group activities, create game rules to meet fan needs, and offer stars marketing channels to be seen and to maintain fan engagement and popularity. Stars, as symbolic products, enable fans to complete self-identification and emotional satisfaction during star chasing, creating traffic and loyal users.

In the Social Symptoms behind the Fan Group Culture, Hu Yong and Liu Chunyi, after studying the communication mode and inner-group operation mechanism, proposed fan group culture has great commercial value. A set of operation logic and business model that is spread from bottom to top as well as from inside to outside, is formed.[5] Combined with their research on the fan group cultural media and communication, the paper concludes that in the relationship mentioned above, the three 
parties create mutual benefits. From the perspective of fans, most of profits models are self-operation. Individual fans or fan groups are the producers and sellers. In the process of "promotion-outputproduction-sell", the fans in the same group serve as major consumers, stars as producing materials, and platforms as channels of marketing and promotion. From the perspective of platforms, the profit model is divided into three categories. The first is to realize its own commercial value on the basis of user volume and activity to receive investment and financing. The second is to earn platform fees and profit from user consumption based on the cooperative marketing between the platform and stars. The third is to sell its own products. For example, social media platforms sell their functions such as VIP service and advertisement, whereas e-commerce platforms make profits from taking a cut of sales and merchant entry fee. From the perspective of stars, they profit from improving their business value to have more business resources on the basis of considerable traffic that is gained from platform promotion and attracting more fans.

\subsection{The Strategy Study of the Tv Drama Marketing and Promotion on Weibo in the Domestic Academia}

As an important part of social media in the new media era, the unique information communication mode of Weibo shows strong marketing and promotion ability, which is recognized by all parties. Therefore, all drama producers regard Weibo marketing as a key link in the integrated marketing.

\subsubsection{Multi-agent Media Promotion Matrix}

According to the Tan and Wu's Research on the Marketing System and Logic of the TV Drama in the Name of People on Weibo [6] and Li's Research on the Marketing of the TV Drama The Story of Minglan on Weibo [7], the Multi-agent media communication matrix refers to the integration strategy of the integrated communication mode, made up of the drama official account, actor accounts, platform official account, and we-media accounts. The four parties implement a two-path influence mechanism. The official account and platform official account, serving as a center path, can persuade consumers through content sharing in three stages, before, during and after the broadcast of the TV drama. At the same time, actor accounts and we-media accounts, serving as opinion leaders, attract consumers from the edge path through the guiding contents containing two aspects, product attention and product attitude, such as calls and comments.

\subsubsection{The Agenda of the Weibo Marketing}

The academia applies the Agenda Setting Theory to the specific research of Weibo marketing and puts forward the marketing subjects deliver the content at the relevant key time nodes to attract the audience. Specifically, before the broadcast, media subjects report the TV drama production progress to inform the fans and interested users of the viewing time. During the broadcast, they produce relevant posts such as drama guides and key plot updates to attract the audience to continuously watch and discuss. After the broadcast, the after service, including the latest news of the TV drama and heated discussion, providing guidance for potential audience and the audience who are used to watch dramas finished.

\subsubsection{Multi-dimensional Marketing}

Liu (2018) analyzed the marketing of the TV drama White Deer Plain on Weibo. He proposed that the effective promotion of White Deer Plain consists of activities such as stickers production based on CP topics, audience promotion, and content themes.[8] Based on the research of other scholars, the author concludes the integrated marketing into four dimensions, namely activity, topic, emotion, and content. Activity marketing refers to using interactive activities such as lottery to motivate users engagement. Topic marketing attracts potential consumers to watch the TV drama through updating hot topics. Emotion marketing tries to appeal audience emotions through conveying emotions of special significance such as nostalgia. Content marketing means the the multi-directional marketing of drama materials containing interesting sidelights. 


\subsubsection{Problems in the Marketing and Promotion Strategy}

According to Su and Jiang's Research on the Strategies and Innovations of TV Dramas' Official Micro-blog Marketing [9], Xu Yao's Research on the Marketing Strategy of Domestic TV Drama Official Accounts on Weibo [10], and the research of other scholars, the paper summarizes three major problems of the current TV drama Weibo marketing consisting of assembly line operation, excessive entertainment, and lack of innovation. As the marketing power of Weibo is attached great importance to, all TV dramas will choose to adopt it. But most of the marketing activities do not grasp the essence of audience participation and ignore the continued efforts in the post-promotion, leading to the difficulty of secondary heated discussion and industrial chain extension.

Combing and summarizing the above research related to the Weibo marketing of BL drama adaptations, the author finds that although the current domestic research on the Weibo marketing of TV dramas has its own emphasis, the common analysis framework and landing points can be summarized into three aspects: the strategy, existing problems and influence, and improvement measures. In other words, there is still room for improvement in the detailed research of the Weibo marketing of TV dramas because there is no complete and in-depth research system of Weibo marketing and the application of quantitative research methods is also relatively weak.

In conclusion, with the quantitative research method of content analysis, the paper analyzes the marketing activities of the representative BL drama adaptations, makes observations while the event is still in the development, and tries to achieve breakthroughs in the marketing strategy of BL drama adaptations, providing experience for other scholars. In the mean time, the paper will comprehensively consider the fields that fans may influence in TV drama marketing and interpret the Weibo marketing and promotion of BL drama adaptations from the perspective of BL adaptation subculture, content production, and traffic monetization, trying to innovatively supplement the academic gap of TV drama Weibo marketing research in China.

\section{Research Design}

\subsection{Research Method and Sample Selection}

Targeted at Weibo, a platform mainly depending on fan economy, the research selects the relevant accounts, such as the official account and the super group, of the hot BL drama Guardian as well as the super group of The Untamed to do case analysis with the method of quantitative content analysis. Taking the posts related to the two TV series as study subjects, the author explores the post content and analyzes the influence of fan culture on drama marketing in the context of fan economy.

\subsection{Analysis Categories and Units}

After browsing the posts related to drama marketing, Guardian, which started BL drama adaptation in 2018, and The Untamed, which made BL drama adaptations favored by the public in 2019, are selected to be the research models. Since Guardian aired (March 2017-January 2021), there have been $800 \mathrm{k}+$ posts on Weibo sent by the media accounts related to Guardian, including @ Guardian (925k followers), @Zhu Yilong Studio (1.51m followers), @Bai Yu Studio (388k followers), and the super group Guardian (985k followers); 480k+ posts sent by the media accounts related to The Untamed consisted of @The Untamed Official (4.645 followers) and the super group The Untamed $(2.187 \mathrm{~m}$ followers). From the perspective of fans, star officials, and TV dramas, the paper is going to analyze post content from the following seven categories with reference to the previous research structures and TV drama marketing features.

1. Post Types: content sharing, Re-creation, role promotion, achievement sharing, and life sharing

2. Presentation Forms: texts, pictures and tests, and videos

3. Styles and Unique Features: funny, lyrical, and rational

4. Popularity: re-post, like, and comment 
5. Account Categories: entertainment bloggers, film and drama bloggers, personal accounts, and others

6. The Number of Fans: less than 10k, 10k-100k, and 100k+

7. Key Words: emotions, drama lines, acquaintance and company, hero names, lottery draw, and support

\section{Analysis of the Influence of Fan Culture on the TV Drama Marketing from the Perspective of Fan Economy}

\subsection{Analysis of Basic Situation}

\subsubsection{Types of Weibo Posts: Mainly Re-creation and Role Promotion}

In accordance with the hashtag presentation rules of Weibo super group, that author randomly samples 1000 posts almost covering the period from 2017 to 2021 based on the latest reply time. Among them, there are 840 effective posts of the hashtag Guardian and 825 effective ones of the hashtag of The Untamed. Based on the sampling results, there are five categories of the posts in the two super groups, namely content sharing, Re-creation, role promotion, achievement sharing, and life sharing.

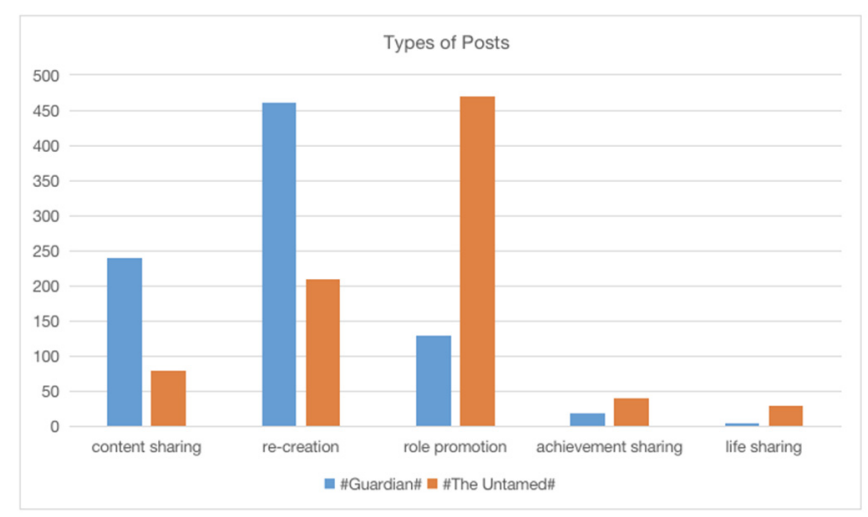

Fig 1. Types of posts

As the table shows, for Guardian, the posts of Re-creation (55\%) account for the most, the ones of role promotion and achievement sharing (1\%) the least. And for The Untamed, the posts of role promotion (57\%) account for the most. In conclusion, both the two super groups involve five types of posts, but the posts of Guardian are mainly Re-creation ones while The Untamed role promotion ones.

The fan group of Guardian focuses more on the TV drama and protagonists. Their affection expression and inner-group communication goes through recreations such as cartoons and fan-fictions, content sharing, and life sharing. Weifen (fans that love one star only), CPfen (fans that ship a screen couple), and drama fans. For The Untamed, fans tend to focus more on the star. They build fan group through Re-creation such as the mix-editing of stars' personal videos and role promotion of the design and modeling. Weifen takes the major pat in the fan structure.

Fron the perspective of TV communication influence, the super group Guardian, based on the content of the drama, promotes the TV series with a boy's love core, attracting the audience mainly consisting of drama fans, BL fans, and star fans. The super group The Untamed, with roles as the carrier, promotes the TV series through star influence, attracting the audience that mainly contains star fans. 


\subsubsection{Pictures and Texts based on Emotions Enjoy a Higher Popularity}

Through the cross analysis of expression form and style characteristics, it can be concluded that the posts of both Guardian and The Untamed are mainly lyrical pictures, amounting to 500+. And the video and pure text posts account for less than $20 \%$. The difference between the two super groups mainly lies in that the number of funny or rational posts of Guardian are more than that of The Untamed whose lyrical posts account for up to $89 \%$.

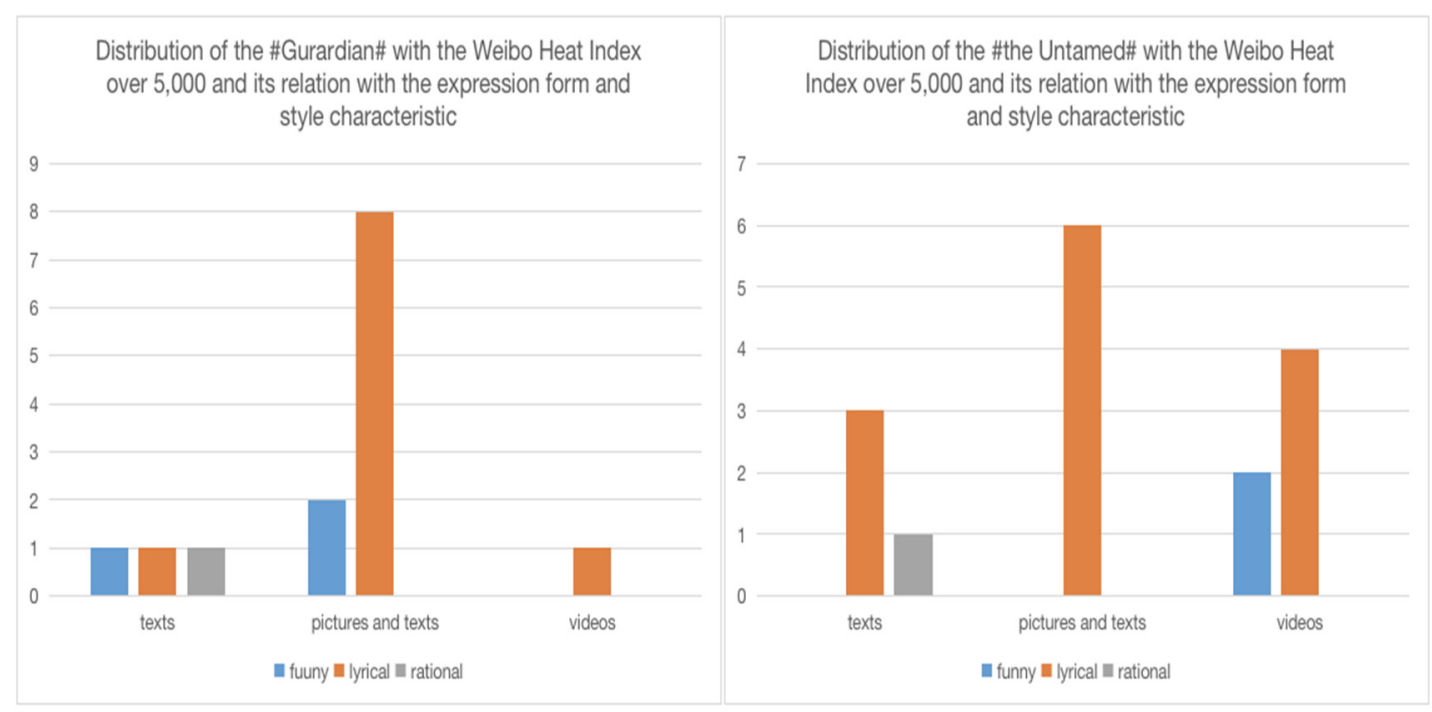

Fig 2. Post comparison between \#Guardian\# and \#The Untamed\#

The author selects the posts with over 5000 comments to analyze the characteristics and popularity. The findings are as follows. There are 14 posts of Guardian with 5000+ re-posts, comments and likes, lyrical ones accounting for $57 \%$. The most popular one is an official lottery post with $839 \mathrm{k}$ re-posts, comments, and likes. For The Untamed, there are 16 posts with $5000+$ re-posts, comments and likes, lyrical pictures $(37.5 \%)$ and videos $(25 \%)$ accounting for the most. The best liked one is an official statement with $87 \mathrm{k}$ re-posts, comments, and likes.

As the main way of content production, emotion is the core of the unity within the fan groups of Guardian and The Untamed. Weibo, as a media, provides a platform for fans to act in group. In the meantime, it also creates game rules, collects and spreads relevant information to meet the needs of fans maintaining the stickiness fans and the popularity of the TV drama. On the other hand, the information including topic popularity and discussion volume can help to promote the TV drama and celebrities effectively and become one of the visual data of their traffic monetization.

\subsubsection{Influence of Account Type and Follower Number on the Traffic of Weibo}
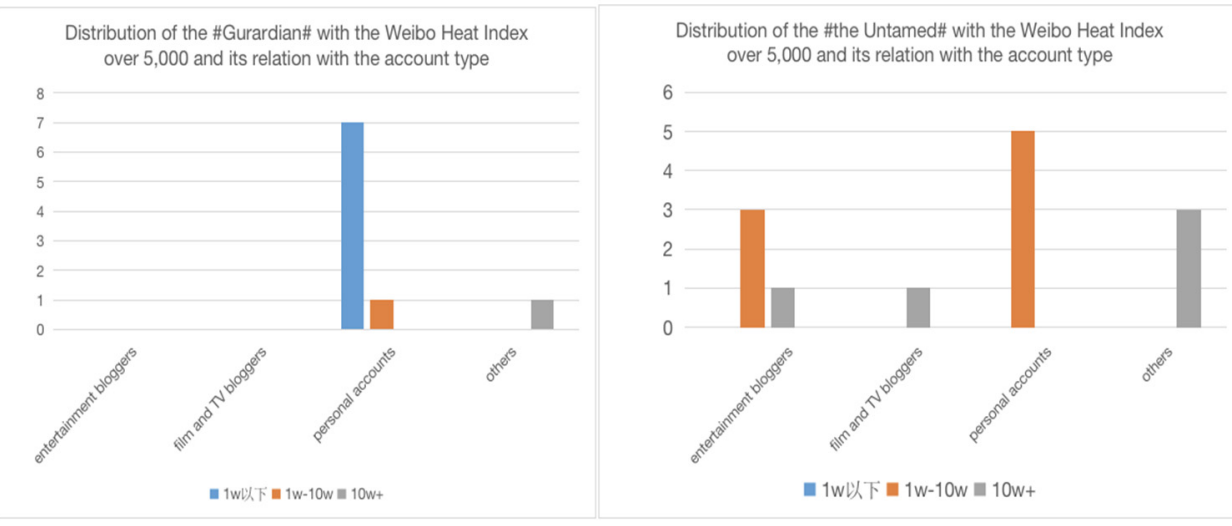

Fig 3. Distribution comparison between \#Guardian\# and \#The Untamed\# 
It has been found that the type of account and the scale of followers have less influence on the Weibo heat index (an indicator showing the popularity of each hashtag) of \#Guardian\# on Weibo and greater influence on \#the Untamed\#.

After sorting out the Weibo accounts, this paper selected four major types of accounts (entertainment bloggers, film and TV bloggers, personal accounts, and others) and three kinds of follower volume (fewer than $1 \mathrm{w}, 1 \mathrm{w}-10 \mathrm{w}$, and $10 \mathrm{w}^{+}$, and $\mathrm{w}$ in the context of Chinese, represents $10,000)$ for the analysis of Weibo Super Group.

As shown in the two Figures, posts about of \#Guardian\# on Weibo with the heat index over 5,000 are all from personal accounts apart from one sent by the official account of the TV drama. Among them, there are 7 bloggers with fewer than $1 \mathrm{w}$ followers and 1 blogger with 1w-10w bloggers; By contrast, posts about \#the Untamed\# with the heat index over 5000 are mostly sent by personal accounts and entertainment bloggers, while there are some belong to the type of film and television bloggers, and others (the official account, 1reading blogger, and 1 photography blogger). Moreover, the accounts enjoying quite high heat index all own more than $1 \mathrm{w}+$ followers, of which there are four with $10 \mathrm{w}+$ followers.

In \#Guardian\#, the heat index is mainly determined by content, while in \#the Untamed\#, it is more obviously related to the volume of followers of the bloggers, and content is not a decisive factor. This is related to the fan base and the development period of BL TV drama. \#Guardian\# opened a new chapter - a time of BL drama. There were few Weibo bloggers expertise in this field at that time, and most of the discussions in super talk were initiated by personal accounts with diverse content and low homogeneity. Therefore, fans tended to choose by preference. Different from \#Guardian\#, \#the Untamed\# was released during the heyday of BL drama in the public eye, and there were more bloggers expertise in this field participating in this field as KOLs to guide the content trend and fans' choice. In this case the super group demonstrated high homogeneity, so fans tended to participate in the discussion of certain posts sent by professional bloggers.

\subsubsection{The Relationship between Keywords and Weibo Heat Index}

Statistics shows that content related to love and including the name of the male and female protagonist gains higher popularity.

The keywords of the two super groups were divided into six categories: love, lines in the drama, encounter and company, the name of the male protagonist, lucky draw, and support. In \#Guardian\#, "lines in the drama" and "love" each accounted for 30\%, and "encounter and company" for $25 \%$. In \#the Untamed\#, "the name of the protagonists" took up 50\%, while "encounter and company" and "lines in the drama" respectively accounted for $20 \%$.

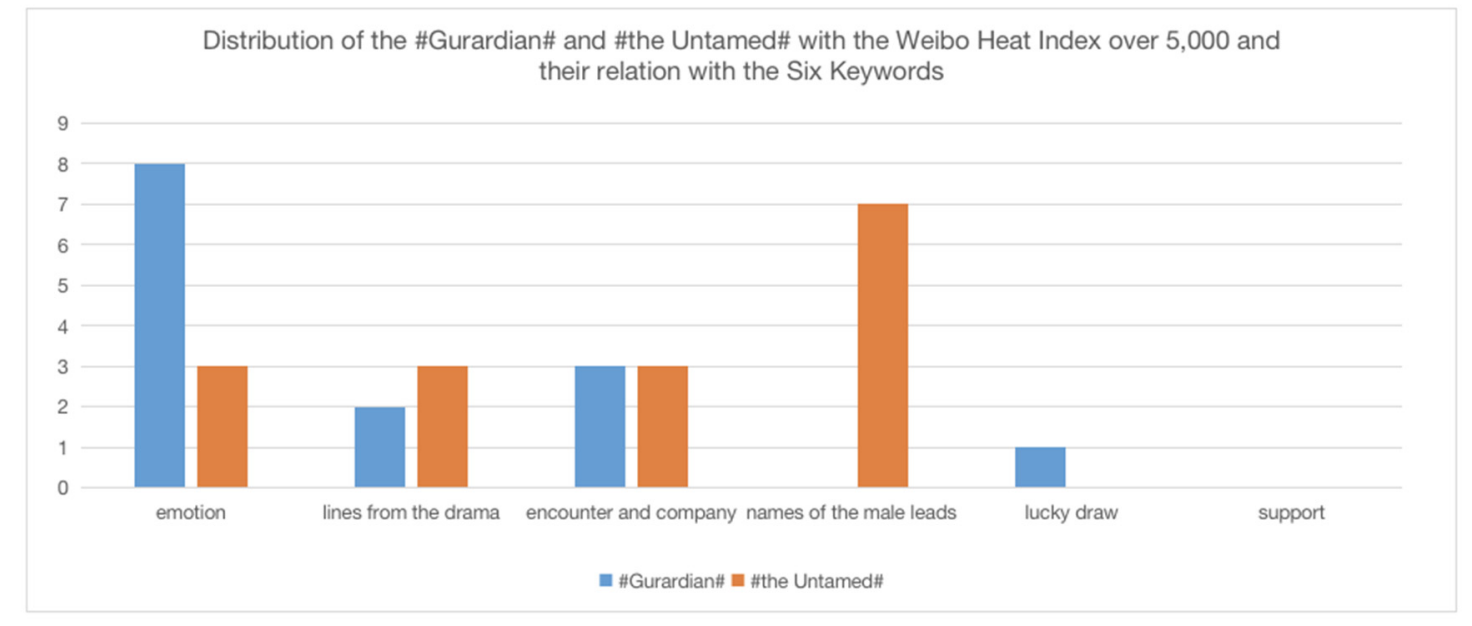

Fig 4. Distribution of the \#Guardian\# and \#The Untamed\# with the weibo heat index over 5000 and their relation with the six keywords 
Posts about \#Guardian\# containing the keyword "emotion" were more popular, while posts about \#the Untamed\# containing the names of the male leads were more popular. Keywords "lucky draw" and "support" were generally less popular in both super groups, while "encounter and company" and "lines from the drama" were frequently-seen and hot. This indicates that \#Guardian\# promote themselves more effectively by presenting the emotion between the two male leads while \#the Untamed\# gained more popularity by the star casts.

When combining the analysis of the relationship between keywords and heat index combined with the percentage of post types, it can be concluded two dramas are different from each other group in terms of the fan group composition and the fans connection. The fan group of \#Guardian\# is built based on their interest and discussion on various aspects including the character, plot and the lead actors while the fan group of \#the Untamed\# is formed based on the both their love for the casts and the plot.

\subsection{Analysis on the Marketing and Promotion Approaches of BL Drama}

\subsubsection{Guardian}

\subsubsection{Stars' Studio}

Before and after the release of Guardian, the Weibo account (@Zhu Yilong's Studio) and (@Baiyu's Studio) respectively released 83 and 73 posts related to it, among which the post types are mainly in 3 categories, CP promotion, the image of the guardian, and personal events. The functions can be divided in 4 types, building persona, promoting the roles, event sharing, and agenda setting.

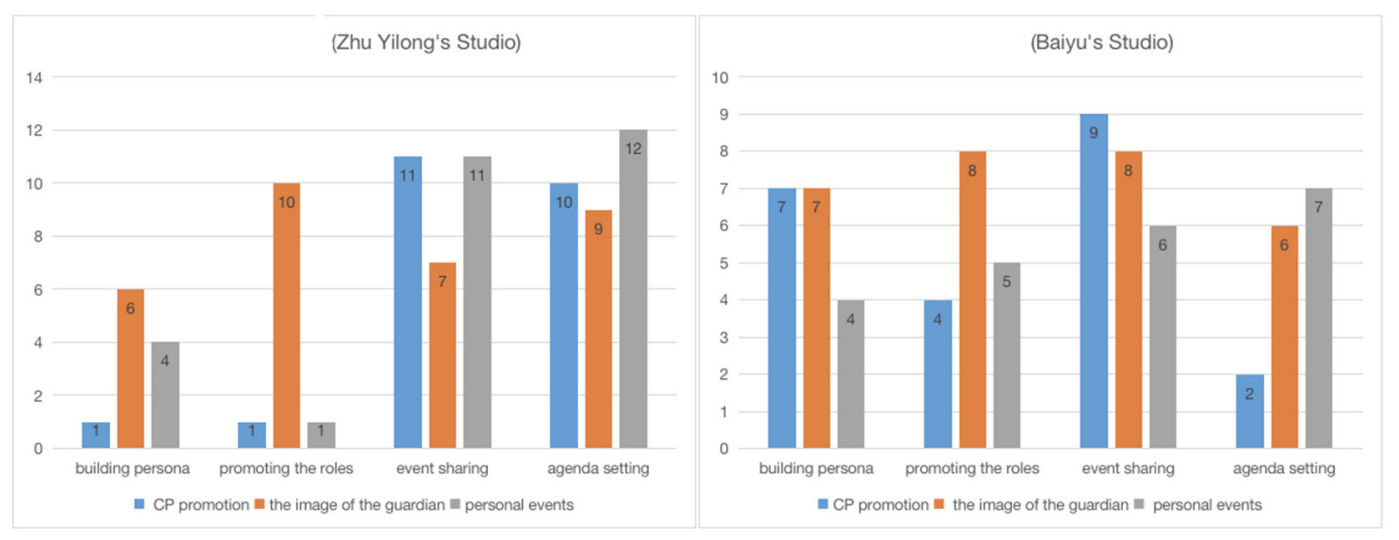

Fig 5. Comparison of content announced by two star studios

It can be found that the marketing and promotion approaches of the two studios are designed to conform to the characteristics of the fan group. This not only helps them to satisfy the fans' need and attracted millions of viewers for the Guardian, but also is conducive to enlarging the fan group of Zhu Yilong and Bai $\mathrm{Yu}$. Besides, their promotion has caught fans' special taste for the emotion between the two protagonists. Consequently, Zhu and Bai successfully gained much clout in 2018 and the BL drama paved its road for future development.

\subsubsection{The Official Account of the Guardian on Weibo}

From March 2017 to August 2018, the official account of the Guardian published 510 posts in total, which can be divided into 5 types, namely, the interaction with fans (voting, lucky draw and live show), the interaction with other stars (launch, birthday blessing, completion of the shooting, etc.), events sharing (announcement and achievement), content sharing (plots and interesting sidelights), and CP promotion (interview with the two leads, bonus scene and plots about the two leads only). 


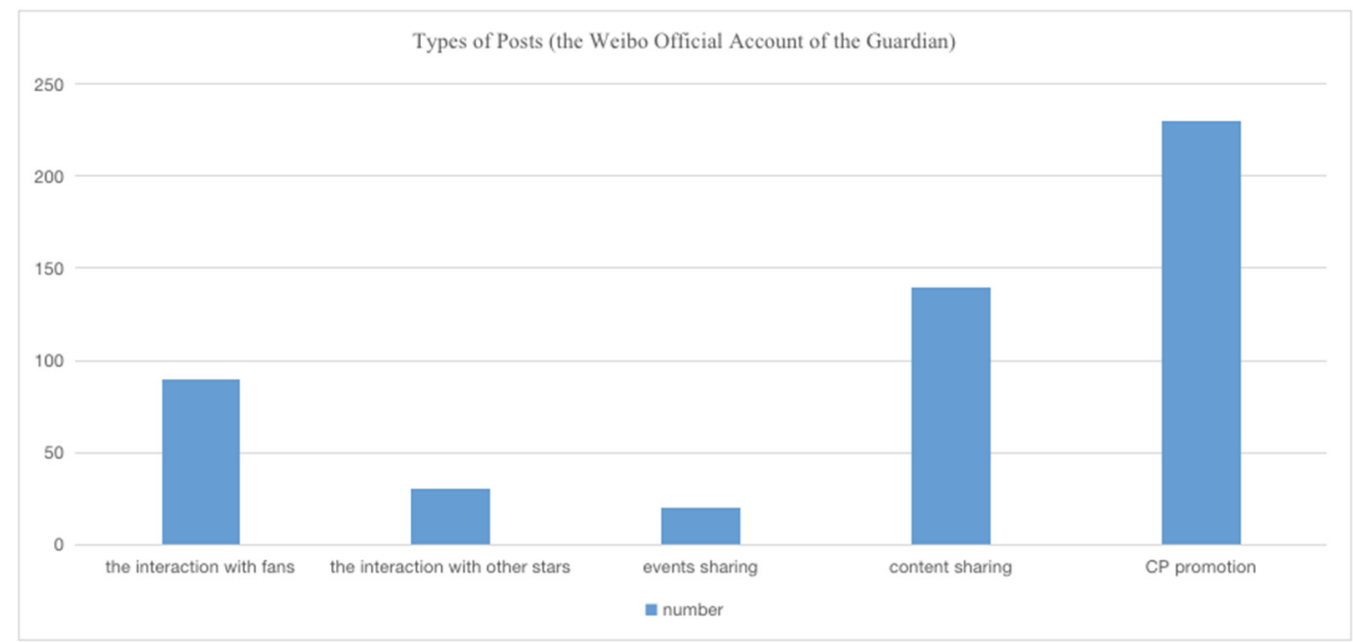

Fig 6. Types of Posts (the Weibo Official Account of the Guardian)

Among them, there are more than 230 pieces about the CP promoting, which accounts for more than $45 \%$ of all, the content sharing (over 140 pieces) for $27 \%$. These two are the major content. Posts about the interaction with fans reaches more than 90 , the interaction with other stars over 30 , and the events sharing over 20.

The Guardian mainly marketed and promoted itself by the CP content. Meanwhile, it sent posts quite frequently to sustain its heat index on Weibo and announce its schedule in time. Besides, it launched lucky draw activity and solicited posts and articles with awards to ensure it can gained followers and popularity continuously. In combination with the analysis of the fan group image, the official Weibo account adopted marketing and promotion approaches suitable for the fans of different types (mainly fans for the TV plot and for the CP) and Guardian had, by the time of its last episode, achieved view counts as high as 2.7 billion in total and the view counts per day were among the top 3. The highest daily view counts were over 100 million. Besides, its focus on BL made the hashtag \#Guardian\# receive the number of page views surpassing 11.04 billion and the discussion volume over 167.44 billion. This hashtag ranked $1^{\text {st }}$ on a lot of real-time lists including the super group and online drama. With tags related to it appearing on Weibo hot search for over 120, it paved the road of BL era.

\subsubsection{The Marketing and Promotion Approaches of the Untamed}

\subsubsection{Weibo Official Account of the Untamed}

From April 2018 to June 2020, the Weibo official account of the Untamed released 457 posts, which can be divided into 5 types as the above.

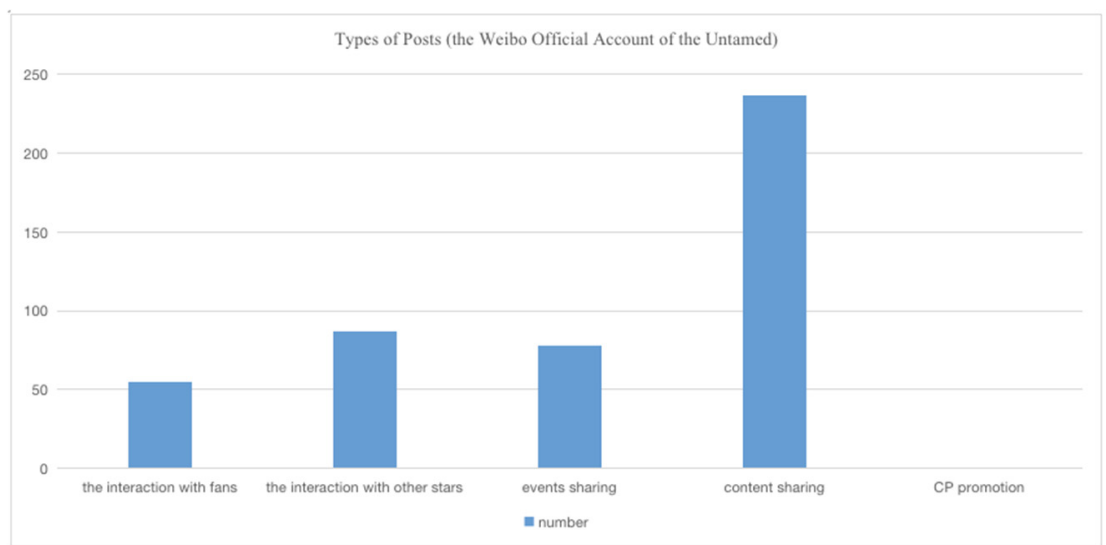

Fig 7. Types of posts (the weibo official account of the Untamed) 
Volume 17 (2022)

Among them, the majority is about the content sharing (more than 240 pieces), which accounts for more than $52 \%$ of all. Posts about the interaction with fans reaches more than 50 , the interaction with other stars over 90 , and the events sharing over 80 . Interestingly, there are few about the CP promotion.

The Untamed mainly promoted itself by content sharing and maintained a high release frequency. It also attracted popularity through various blog types such as event sharing, interaction with the creation team, lucky draw, soliciting articles, concert and new derivatives' on. When the analysis of the fan group characteristics is also taken into account, it can be seen that the official Weibo marketing and communication means of the TV series coincided with the characteristics of both drama fans and CP fans, meeting the needs of fans. It reached the achievement of 10 billion views in 2021; at the same time, it matched the preference of fan groups for individual celebrities, gaining a very high degree of popularity by sharing the stories of multiple creators, parting speeches and other forms of celebrity interaction. Besides, adapted from a BL creation, this drama made both the protagonists and foils famous and gained a large volume of traffic online, capturing much attention and investment and enlarged the market of BL drama.

\subsubsection{Early Access Service and Concert}

Early access is a value-added service launched by online video sites, where paying additional fees on top of membership can unlock episode content in advance. The Untamed released in August 2019 was the first drama providing this service. According to incomplete media statistics, Tencent Video earned more than 75 million yuan on the first day of the service subscription. This is somewhat correlated with the group characteristics of \#the Untamed fans' preference for individual stars. Fans' love for stars prompts their willingness to create good data for stars in the form of consumption, and at the same time, they can accomplish self-identification and emotional satisfaction in the process of following stars. This is one of the fundamental reasons why the early success service can be successful in marketing.

In November 2019, the Special Concert for the Untamed with Ancient Chinese Characteristics were held in Nanjing for two consecutive sessions, with tickets sold out in 5 seconds for a maximum of 1,980 yuan and a minimum of 627 yuan on the opening day of the concerts, and scalpers also once bid up to 150,000 yuan a ticket. In addition to the amazing number of tickets sold offline, the online concert also required a fee of 30 yuan (50 yuan for non-VIP) to get "electronic tickets" to watch the two concerts live simultaneously. By the end of the live broadcast, the number of viewers of the online live broadcast had reached 3.267 million, and the broadcast platform earned nearly 100 million yuan from the online live broadcast alone, which was a new model of profitability in the entertainment industry under the stimulation of fan economy. The show and the broadcast platform have well grasped the psychological aspects of the fans and satisfied their sense of ritual as a fan of the Untamed with the emotional demand of "final farewell". According to the analysis of Bakhtin's theory of carnival, the producer provided a two-day "ideal country" for the fan group, so that the fans could immerse themselves in the "shared emotion" with other peers under the instinctive crowd mentality with high interaction.

\section{Discussion and Inspiration of Online Rumors Spread and its Mechanism}

\subsection{Targeted Content Production Oriented to Fans' Needs}

In the era of Internet, fan groups are an important part of today's entertainment industry. With its advantageous features of complete organizational structure, multi-levelness, formality of operation, perfect functionality and large scale, it can provide the organizational basis and conditions for realizing economic benefits of stars. In the fan economy, the brand loyalty of corporate marketing is directly transformed into the emotional attachment of fans to the stars. In the organized operation and "group carnival", fans are more consciously and fanatically stimulated to invest their money and time in things related to the stars, and achieve a considerable amount of data through consumption. However, the consumption and profitability of fan groups are based on the premise that the stars they 
chase after meet their needs. As the data analyzed above shows, the TV series "Guardian" and "the Untamed" have undoubtedly been successful in content production and marketing.

The mode and process adopted by the two series on Weibo for promotion are basically the same as the other TV series has. Weibo official accounts and celebrities cooperate with each other, introducing TV series topics to build momentum in the early stage and informing fans and interested viewers of the time to watch. During the broadcast period, the drama is combined with previews and key plot updates to attract viewers to continue watching and discussing. After the broadcast, they share the latest news of the TV series and hold after-sales services such as related program show. The whole process is combined with a Multi-dimensional marketing mix of activities, topics, emotions and content, such as lucky draws and solicitations, to open up the market and maintain the popularity. However, in the same model, "Guardian" and "the Untamed" gained more popularity. This is based on the fact that the three participants of the two TV series, the fan base, the creation team, and the star studio, are linked to each other, the basic direction fits together, and the marketing and promotion activities are targeted.

The creation team and celebrity sides analyze the group portraits based on the performance of fans in Super Groups, identify the preferences and needs of fan groups, and produce targeted content based on their characteristics. The fan groups show more obvious attention and consumption behavior under the matching content. In the current era of fan economy, TV series and celebrities should uphold the concept of "audience orientation", pay attention to the group portrait analysis of fans, use big data, cloud computing and other Internet technologies to collect market and audience information through account types, Super Groups and hot search data, explore the preferences and needs of fans, and produce targeted content in a reasonable and standardized manner. In order to achieve the purpose of efficient marketing and propaganda, the content production should be targeted in a reasonable and standardized manner.

\subsection{Based on the Emotion Economy of the Fan Group, Exploring Diversified Traffic Monetization}

\subsubsection{Representation of "Cultural Industrialism"--Emotional Economy of the Fan Group}

With the cultural industry becoming increasingly advanced, social media developing and fan culture flourishing, the formerly loose and independent individuals in the fan culture are linked with each other in new channels and continue to develop into the "Fan group", one of the subcultural groups, which is discussed in this paper.

Fans in the tightly structured fan group are no longer confined by old-fashioned systems, but begin to strengthen their ties and interactions, engage in group normative behaviors such as content production and data operation, and to some extent become a kind of emotional economy. The core is the voluntary payment behavior of fans. This emotional consumption has weakened the consideration of many factors such as the commodity itself (including the stars) when fans are consumers, and strengthened the emotional extension. Under this phenomenon, the capital side has created a new cultural industry with platform as the structure, data as the currency, and fans who perform emotional labor as digital labor, forming a new economic model.

The TV series "Guardian" takes advantage of the fan group's preference for the emotions of the double male leads for marketing, calling for votes, soliciting ideas, and so on to generate direct profits. It also boosts the advertising revenue of "Guardian" through fan data and discussion volume, and sells a series of derivatives to gain secondary profits. By the final day, it achieved 2.7 billion total plays, the top three consecutive daily plays, and the highest single-day play of 100 million, which unlocked the era of adapting BL creation into drama and created a way to gain profit after the IP.

\subsubsection{Exploration of Diversified Means of Traffic Monetization}

The TV series "the Untamed" has created a new after-sales service(concert) of TV series based on "Guardian", which has somehow extended the life cycle of TV series and created a new way of making profit for the creation team and stars. According to the formal data, the concert tickets were 
sold out in 5 seconds, and the number of people who grabbed tickets online was as high as 2.08 million. Since too many people did not get tickets, the concert tickets were touted and the price was even as high as 150,000 yuan.

In addition to the concert, the "Album of the Untamed with Ancient Chinese Characteristics" is still sold at a price of 20 yuan a piece in QQ Music. According to the album page, 1.079 million copies have been sold.

On the day when early access service was on Tencent Video, about 2.35 million netizens purchased the service, and the profit was as much as 60 million yuan. Later on, these service and innovative meetups were mimicked by many TV dramas and became more profitable than ever.

The reason why the two TV dramas have achieved such success is that they are based on the organization structure and operation mode of the fan group, making reasonable use of fan emotions and platform for traffic monetization. Therefore, since consumption is prevailing among fan group currently, the TV series marketing should grasp the core of fan emotion, create TV series data and guide fan production and consumption based on the operation mode of each platform. Furthermore, according to the features of different platforms and the characteristics of fans, the marketing means should be subdivided and more unique and highly compatible with the corresponding platforms, so as to realize more diversified traffic monetization ways.

\subsection{Regulatory Guidance of BL Culture and Dramas Adapted from BL Creations}

Since March 2021, a number of leading companies and actors have entered this field. This is a change from the previous idea which prevents from gaining profits in a less popular field, which marks the emergence of BL culture from subculture to the public. However, the BL drama's prevalence may have an adverse effect on young people who are not able to judge the right from wrong. On September 16, 2021, the National Radio and Television Administration (NRTA) held a meeting in Beijing to resolutely resist the phenomenon of over-entertaining such as the BL culture trend. On the morning of January 6, 2022, the Beijing Radio and Television Bureau said at the national radio and television work exchange meeting that it would stop all $\mathrm{BL}$ dramas. From rising to fame to being called off in less than 3 years, BL dramas are forbidden because of the BL culture and fan group to a large extent.

BL culture has previously been as a minor culture with very few people. This subculture has not had a major impact on mainstream values. However, after the popularization of the BL drama, a lot of BL creation began to be adapted to TV series on a large scale under the joint influence of capital and fans, breaking through the boundary between subculture circle and mainstream idea. The bottomless hype and over-consumption of "BL culture" to meet market demand can have a negative impact on young people.

In terms of the BL culture itself, it always has its own audience, whose aesthetics and needs should be respected. However, it is undeniable that many BL drama are made with the intention of gaining clout by presenting "BL-type" behaviors instead of positively guiding and presenting the culture of BL. Some of the content of these works are not suitable to be viewed by teenage groups whose values are not yet formed. In the face of the differences in groups and needs, the relevant departments should implement grading censorship of such works, which can not only protect teenage groups from being wrongly influenced, but also meet the aesthetic needs of some minor groups. At the same time, "BL drama" should also be regulated, through management and guidance so that it will be standardized as soon as possible. It is necessary to resist gimmicks and hype and the content itself is worth watching.

\section{References}

[1] $\mathrm{Wu} \mathrm{H}$. The Reduction and Countermeasures of the Youth Subculture Communication to the Mainstream Culture[J]. Youth Journalist, 2021(14):103-104.

[2] Fan J J. Examination of the Characteristics and Values of Youth Subculture[J]. People's Tribune, 2021(11):92-94. 
[3] Chen L., Li C. "Neo-tribe" in the Internet Society: A Study of Post-subculture Circle[J]. Media Bserver, 2021(06):5-12.

[4] Ma Z H., Hu L Y. Internesting, Difference and Visibility: New Trend of the Development of Online Youth Subculture[J] Youth Exploration,2021(05):18-27.

[5] Hu Y., Liu C Y. The Social Symptoms behind the Fan Group Culture[J]. Journalistic University, 2021(08):65-79+119.

[6] Tan H H., Wu Y W. Research on the Marketing System and Logic of the TV Drama in the Name of People on Weibo[J]. Journal of Hubei University of Science and Technology, 2018,38(02):134-139.

[7] Li En. Research on the Marketing of the TV Drama The Story of Minglan on Weibo[D]. Hebei University of Economics and Business, 2020.

[8] Liu Q B. Research on TV Drama Marketing Strategy in the Context of New Media[D]. Lanzhou University, 2018.

[9] Su M N., Jiang L X. Research on the Strategies and Innovations of TV Dramas' Official Micro-blog Marketing[J]. Journal of Hunan University (Social Sciences),2017,31(02):142-148.

[10] Xu Y. Research on the Marketing Strategy of Domestic TV Drama Official Accounts on Weibo--Taking the Popular TV Dramas The Story of Yanxi Palace and Ash-like Frost as an Example[J]. Media Form, 2018, 1(12):164-165. 